\title{
Neural Network Compensation Control for Model Uncertainty of Flexible Space Manipulator Based on Hybrid Trajectory
}

\author{
Huang Dengfeng ${ }^{1,2}$ and Huang Xiaoqin ${ }^{3, *}$ \\ ${ }^{1}$ School of Mechanical \& Automotive Engineering, Fujian University of Technology, Fuzhou 350118, China \\ ${ }^{2}$ Fujian Key Laboratory of Automotive Electronics and Electric Drive, Fujian University of Technology, Fuzhou 350118, China \\ ${ }^{3}$ College of Physics and Electronic Information Engineering, Minjiang University, Fuzhou 350108, China
}

Received 29 September 2020; Accepted 14 January 2021

\begin{abstract}
A flexible space manipulator is needed to accomplish various tasks in the space in future. However, model uncertainty and the flexibility of link decrease the trajectory tracking accuracy of a space manipulator. To avoid the influences of model uncertainty and the vibration of flexible manipulator on control accuracy, the trajectory tracking and flexible suppression problems of free-floating flexible space manipulator with an attitude-controlled base were discussed in this work. First, the flexible link was viewed as an Euler-Bernoulli beam. The horizontal deformation of the flexible link was described by the assumed mode method, and a dynamic equation of the flexible space manipulator system was set up by the Lagrange method of the second kind. Second, by considering the model uncertainty of the system, the advantages of the method on the basis of model control were developed and model uncertainty was compensated for by a neural network. Moreover, a neural network compensation control scheme for rigid motion was proposed to achieve coordinated motion between the base's attitude and the joint's angles of flexible space manipulator. Subsequently, a hybrid trajectory that reflects flexible modal and rigid motion simultaneously was designed by using the concept of virtual control force to inhibit flexible vibration actively. A control scheme in line with a hybrid trajectory was proposed. Research results demonstrate that the proposed control scheme can help the base's attitude and two joints' angles of the space manipulator to track the given desired trajectories effectively and also eliminate vibration of flexible links. This study provides an effective scheme to increase control accuracy in the trajectory tracking of the space manipulator under model uncertainty and the vibration of the flexible links in practical conditions.
\end{abstract}

Keywords: Flexible space manipulator, Hybrid trajectory, Neural network, Model uncertainty, Compensation control

\section{Introduction}

With the continuous development and utilization of space resources by humans, space robots are gradually becoming important tools for space activities, a development which has important significance for increasing human ability in conducting the comprehensive exploration, development, and utilization of space. A space robot can assist or replace humans to finish certain heavy and dangerous tasks in outer space, such as carrying, assembly, repair, and production [13]. Therefore, researchers from different countries emphasize the dynamics modeling and trajectory tracking control of space robots [4-6].

The free-floating space manipulator system which has uncontrollable position and controlled attitude of the base can be used from the perspective of saving control fuels. In the microgravity environment of outer space, the position control system of the base is shut down when the space manipulator is in a state of uncontrollable position and controlled attitude of the base. Under this circumstance, the entire system meets the dynamic constraints of conservation of momentum. This situation leads to the strong dynamic coupling effect between the base and the arms. Given that motion of the manipulator can generate a reactive force and

*E-mail address: hxq582p @163.com

ISSN: 1791-2377 @ 2021 School of Science, IHU. All rights reserved. doi:10.25103/jestr.141.09 a moment of reaction to the base, the position and attitude of the base are changed, thereby causing adverse impacts on the trajectory tracking of the space robot. As a result, designing the control system for a free-floating space manipulator becomes more problematic. Moreover, the light slim bar becomes the best choice for the link of the space manipulator in consideration of launch technology and the needs of reducing launch mass to reduce the launch cost. Compared with the rigid space manipulator, a flexible space manipulator system has many advantages, such as large operation space, low energy consumption, high load mass ratio, and compact component design [7-8]. A flexible light space robot can stimulate vibration when it is moving during transport and assembly of space structures, thereby decreasing the manipulation accuracy. Hence, the influences of flexibility of the manipulator link of the space robot system on motion accuracy cannot be ignored [9]. In addition, the flexible space manipulator system has many model uncertainties in practical engineering (such as uncertain kinematic parameters and unknown loads), a feature which complicates the development of an accurate model [10]. During its operation, a space manipulator cannot execute tasks well because of interferences by various factors in outer space. If the influences of model uncertainty are ignored in the design of the controller, then the space manipulator cannot accomplish the regulated operation tasks.

High-accuracy trajectory tracking of a flexible space manipulator must not only resolve interferences and the 
dynamics model uncertainty caused by the dynamic coupling effect, but also inhibit the vibration produced by the flexible link of the manipulator. These requirements increase the difficulties in the design of a control system. On this basis, many academic studies have investigated the dynamic control of space manipulators. However, relevant researches mainly focus on the influences of model uncertainty or the flexibility of links on control performances. Few articles have considered the collaborative effects of model uncertainty and vibration on the controlling performances of the flexible manipulator. Therefore, studying the trajectory tracking control of a flexible space manipulator with model uncertainty has important theoretical values and practical significance. In this study, the flexibility of the manipulator link and the system modeling uncertainty were analyzed and a dynamics model of the flexible space manipulator was established. Moreover, the model uncertainty compensation control scheme and flexible vibration suppression technology were explored thoroughly. Relevant conclusions are expected to provide references to increase the trajectory tracking accuracy of the flexible space manipulator.

\section{State of The Art}

To address the trajectory tracking control of the flexible space manipulator, scholars have proposed many methods to inhibit flexible vibration [11-12]. Some researchers suggested the inhibition of the vibration of the flexible manipulator using devices like the piezoelectric (PZT) actuator and sensor. Lou et al. developed a dynamics model of the space flexible manipulator on the basis of finite element theory by considering the roles of the PZT torsion actuator and PZT shear actuator as well as the tip mass. In line with the modal equation, a linear quadratic regulator (LQR) control was designed to inhibit the bending-torsional coupling vibration of the space double-connecting links [13]. Sabatini et al. studied and designed an active vibration suppression strategy and relevant devices to decrease the structural vibration of a flexible space manipulator during on-orbit operation and proposed an adaptive vibration control program based on a piezoelectric device. They revealed that the quantity, layout, and working mode of piezoelectric devices should be chosen accurately for the convenience of realizing optimal performances and decreasing the elastic oscillation and power consumption under use [14]. Zhang et al. studied a rigid-flexible parallel manipulator with three intelligent links. As each intelligent link contains three piezoelectric sensor and actuator pairs, this manipulator was equipped with self-perception and selfcontrol and can effectively inhibit the vibration caused by the flexibility of links under high-speed working conditions. On this basis, a vibration control scheme based on the adaptive sliding mode method was proposed to attenuate residual vibration in modal space [15]. However, these control schemes all require extra piezoelectric devices, thereby increasing the hardware cost of the control system.

To address the active suppression of flexible vibration, a singular perturbation is a common method preferred by scholars in studying the control of a ground fixed flexible robot. The main idea of a singular perturbation is to decompose the dynamic system of a flexible manipulator into two relatively independent parts. One part is the slow subsystem corresponding to rigid motion, and the other part is the fast subsystem corresponding to flexible vibration. Subsequently, the controllers of these two subsystems are designed independently. Recently, many researchers have applied the singular perturbation into the control of the vibration suppression of the flexible space manipulator. By using the singular perturbation method, Masoudi et al. decomposed the dynamic equation into the zero-order equation which is expressed as the rigid motion of the space robot and the first-order equation that expresses elastic vibration and rigid perturbation. The torque needed for the rigid manipulation of the space robot was determined by using the inverse dynamic method and through an open-loop control scheme, and the digital LQR feedback control scheme was applied to suppress vibration [16]. Zhang et al. proposed a nonlinear partial differential equation observer which estimated the position and speed of the flexible link and implemented dynamic decoupling of the rigid-flexible coupling system through the singular perturbation method for the convenience of controller design. On this basis, a boundary layer control scheme was proposed to adjust the end effector so as to move along the reference trajectory motion in the task space and suppress vibration [17]. Using the singular perturbation method, Yang et al. deduced a composite control scheme that combines backstepping control and active vibration control to track the expected trajectory. The proposed control scheme could realize the closed loop stability of the flexible space robot [18]. Yang et al. deduced an adaptive sliding-mode control to the slow subsystem with unknown but bounded disturbances. Meanwhile, an optimal LQR method for the fast subsystem was suggested to attenuate the vibration of the flexible manipulator [19]. Su et al. disclosed the mutual relations between two adjacent links through recursive kinematics and dynamics and calculated the nominal value of the interaction force as a compensation, with the aim of increasing the tracking accuracy. On this basis, a dispersed adaptive robust controller was designed and a steering logic device was constructed to determine the torque on the joint motors [20]. This combined control scheme based on singular perturbation method directly superposes the rigid motion control quantity and flexible vibration suppression quantity. The said technique can actively inhibit the vibration of flexible links but can also decrease the robustness and performances of the rigid motion controller to some extent.

The effects of model uncertainty must be considered in studying the high-accuracy control of the space manipulator. Therefore, inhibiting or eliminating the influences of model uncertainty on the control of the space manipulator system by choosing an appropriate compensation method has very practical significance. Some scholars have estimated and approximated model uncertainty through robust, adaptive, or neural network methods. Cetin et al. proposed a robust adaptive controller to compensate for the uncertainty of kinetic parameters to assure stable tracking of the end effector of a ground fixed robot [21]. Kumar et al. compensated for an uncertain part in the dynamics model by using a radial basis function (RBF) neural network and realized the asymptotic stability control of a rigid space robot [22]. However, these two studies only focus on a ground fixed or rigid space robot. $\mathrm{Yu}$ et al. designed a reduced order controller composed of one rigid control unit and two flexible control units according to the separation of two-time scales of manipulator dynamics and constructed an augment robust rigid control scheme and an optimal LQR controller to suppress the vibration of a flexible link on the basis of the boundaries of model uncertainty [23]. By considering model uncertainty and external disturbance and eliminating the buffeting phenomenon, Feng et al. proposed 
a robust sliding mode controller by replacing the discontinuous part in the control signals of the RBF neural network to solve the trajectory tracking problem of a rigid space manipulator in task space [24]. Yu et al. studied a six degrees of freedom (6-DOF) space robot which carried a flexible panel, and they proposed a robust adaptive controller in consideration of system uncertainty on the basis of the torque calculation method to eliminate the elastic vibration of panels. Moreover, the stability of the controller was guaranteed by integrating the Lyapunov stability theory [25]. However, designing the control system becomes more difficult because the above robust control schemes for system model uncertainty require a linear function relationship between the dynamic equation of system and inertial parameters.

For the flexible space manipulator system with model uncertainty, by taking full advantage of the existing nominal model of the system and using the model-based control method, that uncertainty was compensated for by a neural network. A neural network compensation control scheme for rigid motion was designed. However, this scheme can finish the trajectory tracking of the coordinated motion of the base's attitude and joint's angles of the flexible space manipulator but cannot control the flexible modal of the flexible link. Hence, the optimal LQR was applied to get the virtual control force. Moreover, a hybrid trajectory that simultaneously reflects the flexible modal and rigid motion was generated with the second-order command generator by using the virtual control force. This reconstructed the original scheme and designed a neural network compensation control scheme on the basis of the hybrid trajectory. The numerical simulation results verified the effectiveness of the control scheme.

The reminder this study is organized as follows. Section 3 describes the dynamics modeling of the flexible space manipulator and the design of a neural network compensation control scheme of rigid motion. This method is improved into a hybrid trajectory control scheme that can simultaneously reflect flexible modal and rigid motion. Section 4 provides a simulation analysis on the trajectory tracking and flexible vibration suppression for the flexible space manipulator with model uncertainty. Section 5 summarizes the conclusions of this work.

\section{Methodology}

\subsection{System dynamics model}

Without loss of generality, a free-floating flexible space manipulator system which generates planar motion was considered. The structure of this system is shown in Fig.1. Suppose that this system consists of a free-floating base $\left(B_{0}\right)$, a rigid link $\left(B_{1}\right)$, and a flexible link $\left(B_{2}\right)$. The body-fixed coordinate systems $\left(O_{i}-x_{i} y_{i}\right)$ of the principal axis of different parts $B_{i}(i=0,1,2)$ are established, where the centroid $\left(O_{C 0}\right)$ of $O_{0}$ and $B_{0}$ overlap. $O_{1}$ and $O_{2}$ are the rotary hinge centers that connect $B_{0}$ and $B_{1}$ as well as connects $B_{1}$ and $B_{2}$, respectively. The $x_{1}$ axis is the symmetric axis of $B_{1} . x_{2}$ is the symmetric axis before the deformation of $B_{2}$ and is always tangential to the flexible link at $\mathrm{O}_{2}$ during the rotation. If the distance between $O_{1}$ and $O_{0}$ on the $x_{0}$ axis is $l_{0}$, then the distance between the centroid of $B_{1}\left(O_{C 1}\right)$ and $O_{1}$ on the $x_{1}$ axis is $a$ and the length of $B_{i}$ along the $x_{i}(i=1,2)$ axis is $l_{i}$. The mass and central inertial tensor of $B_{0}$ and $B_{1}$ are $m_{i}$ and $J_{i}(i=1,2)$, respectively. The linear density of the homogenous flexible link $B_{2}$ is $\rho$ and the bending rigidity is $E I$.

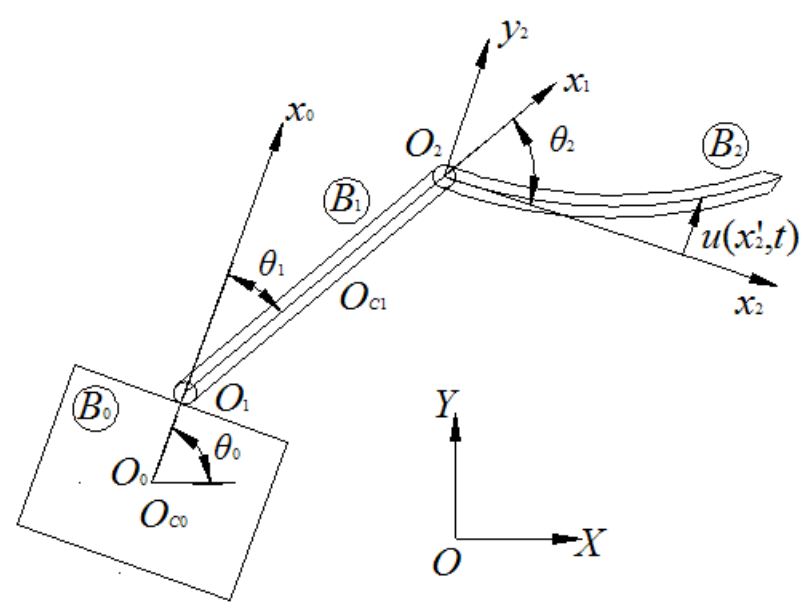

Fig. 1. Free-floating space flexible manipulator system

A translational inertial coordinate system $(O-X Y)$ was set up. Moreover, $\theta_{0}$ is defined as the rotating angle of the $x_{0}$ axis relative to the $Y$ axis. $\theta_{1}$ is the rotating angle of the $x_{1}$ axis relative to the $x_{0}$ axis, and $\theta_{2}$ is the rotating angle of the $x_{2}$ axis relative to the $x_{1}$ axis. If the influences of axial deformation and shear deformation are ignored, then the flexible link $B_{2}$ can be viewed as a Euler-Bernoulli beam. The horizontal deformation of the flexible link is described by the assumed mode method. Let $u\left(x_{2}^{\prime}, t\right)$ be the elastic displacement of the flexible link at the horizontal coordinate $x_{2}^{\prime}$ and $t$ in the vertical direction. Hence, we can obtain

$u\left(x_{2}^{\prime}, t\right)=\sum_{i=1}^{n} \eta_{i}(t) \phi_{i}\left(x_{2}^{\prime}\right), \quad\left(0 \leq x_{2}^{\prime} \leq l_{2}\right)$

where $\eta_{\mathrm{i}}(t)$ is the modal coordinates, $\phi_{i}\left(x_{2}^{\prime}\right)$ is the $i$-order modal function, and $n$ is the truncation term and it is determined the second-order modal, $n=2$.

If the tiny gravity gradient is ignored, then the floating flexible space manipulator system with an attitudecontrolled base is a free-floating rootless multi-body system without external influences. The system observes the conservation of the momentum of $(O-X Y)$. If the relative rotating angle between the base's attitude and the two hinge joints of the floating flexible space manipulator $\left(\boldsymbol{\theta}=\left(\begin{array}{lll}\theta_{0} & \theta_{1} & \theta_{2}\end{array}\right)^{\mathrm{T}}\right)$ and the modal coordinates of the flexible link $\boldsymbol{\eta}=\left(\begin{array}{ll}\eta_{1} & \eta_{2}\end{array}\right)^{\mathrm{T}}$ are used as the generalized coordinates of the system, then the dynamic equation of the system can be gained according to the conservation of momentum and the Lagrange method of the second kind:

$$
\boldsymbol{D}(\boldsymbol{\theta}, \boldsymbol{\eta})\left[\begin{array}{c}
\ddot{\boldsymbol{\theta}} \\
\ddot{\boldsymbol{\eta}}
\end{array}\right]+\boldsymbol{C}(\boldsymbol{\theta}, \dot{\boldsymbol{\theta}}, \boldsymbol{\eta}, \dot{\boldsymbol{\eta}})\left[\begin{array}{c}
\dot{\boldsymbol{\theta}} \\
\dot{\boldsymbol{\eta}}
\end{array}\right]+\left[\begin{array}{c}
0 \\
K \boldsymbol{\eta}
\end{array}\right]=\left[\begin{array}{c}
\boldsymbol{\tau} \\
0
\end{array}\right]
$$

where $\boldsymbol{D}(\boldsymbol{\theta}, \boldsymbol{\eta})$ is the $5 \times 5$ symmetric and positive defined mass matrix. $\boldsymbol{C}(\boldsymbol{\theta}, \dot{\boldsymbol{\theta}}, \boldsymbol{\eta}, \dot{\boldsymbol{\eta}})\left[\begin{array}{ll}\dot{\boldsymbol{\theta}}^{\mathrm{T}} & \dot{\boldsymbol{\eta}}^{\mathrm{T}}\end{array}\right]^{\mathrm{T}}$ is the 5-order column vector that contains the Coriolis and centrifugal forces. $\boldsymbol{K}=\operatorname{diag}\left(k_{1}, k_{2}\right)$ is the rigid matrix of the flexible link, $k_{i}=E I \int_{0}^{l_{2}} \phi_{i}^{\prime \prime} \phi_{i}^{\prime \prime} \mathrm{d} x_{2}^{\prime},(i=1,2) . \quad \tau=\left(\begin{array}{lll}\tau_{0} & \tau_{1} & \tau_{2}\end{array}\right)^{\mathrm{T}}$ is the 3-order column vector which is formed by the control torque of the base's attitude $\left(\tau_{0}\right)$ and the control input torques $\left(\tau_{1}\right.$ and $\left.\tau_{2}\right)$ of the two hinge joints $\left(O_{1}\right.$ and $\left.O_{2}\right)$ of the manipulator. 
3.2 Neural network compensation control of rigid motion A neural network control scheme on the basis of model uncertainty compensation was proposed in this section to achieve the trajectory tracking of the rigid motion of the flexible space manipulator. This scheme is composed of the calculation moment method based on the nominal model of the flexible space manipulator system and the compensation control unit based on the RBF neural network. The compensation control unit improves tracking performance. The neural network was trained according to the Lyapunov theory, an approach which assured convergence of the learning algorithm.

For convenience in the design of the control scheme, the dynamics system of the flexible space manipulator (Eq.[2]) was expressed by block matrixes:

$$
\left[\begin{array}{ll}
\boldsymbol{D}_{r r} & \boldsymbol{D}_{r f} \\
\boldsymbol{D}_{f r} & \boldsymbol{D}_{f f}
\end{array}\right]\left[\begin{array}{l}
\ddot{\boldsymbol{\theta}} \\
\ddot{\boldsymbol{\eta}}
\end{array}\right]+\left[\begin{array}{ll}
\boldsymbol{C}_{r r} & \boldsymbol{C}_{r f} \\
\boldsymbol{C}_{f r} & \boldsymbol{C}_{f f}
\end{array}\right]\left[\begin{array}{c}
\dot{\boldsymbol{\theta}} \\
\dot{\boldsymbol{\eta}}
\end{array}\right]+\left[\begin{array}{c}
0 \\
\boldsymbol{K} \boldsymbol{\eta}
\end{array}\right]=\left[\begin{array}{c}
\boldsymbol{\tau} \\
0
\end{array}\right]
$$

where $\boldsymbol{D}_{r r}$ and $\boldsymbol{C}_{r r}$ are $3 \times 3$ submatrixes. $\boldsymbol{D}_{f f}$ and $\boldsymbol{C}_{f f}$ are $2 \times 2$ submatrixes. $\boldsymbol{D}_{r f}=\boldsymbol{D}_{f r}{ }^{\mathrm{T}}$ is a $3 \times 2$ submatrix. $\boldsymbol{C}_{r f}$ and $\boldsymbol{C}_{f r}$ are the $3 \times 2$ and $2 \times 3$ submatrixes.

Eliminating $\ddot{\boldsymbol{\eta}}$ from Eq.(3) yields

$$
\begin{array}{r}
\boldsymbol{D}_{r r} \ddot{\boldsymbol{\theta}}-\boldsymbol{D}_{r f} \boldsymbol{D}_{f f}^{-1} \boldsymbol{D}_{f r} \ddot{\boldsymbol{\theta}}-\boldsymbol{D}_{r f} \boldsymbol{D}_{f f}^{-1} \boldsymbol{C}_{f r} \dot{\boldsymbol{\theta}}-\boldsymbol{D}_{r f} \boldsymbol{D}_{f f}^{-1} \boldsymbol{C}_{f f} \dot{\boldsymbol{\eta}}- \\
\boldsymbol{D}_{r f} \boldsymbol{D}_{f f}^{-1} \boldsymbol{K} \boldsymbol{\eta}+\boldsymbol{C}_{r r} \dot{\boldsymbol{\theta}}+\boldsymbol{C}_{r f} \dot{\boldsymbol{\eta}}=\boldsymbol{\tau}
\end{array}
$$

Eq.(4) is written in the form similar to the dynamic equation of a rigid manipulator:

$$
\boldsymbol{D}_{r e q}(\boldsymbol{\theta}, \boldsymbol{\eta}) \ddot{\boldsymbol{\theta}}+\boldsymbol{C}_{r e q}(\boldsymbol{\theta}, \boldsymbol{\eta}, \dot{\boldsymbol{\theta}}, \dot{\boldsymbol{\eta}})=\boldsymbol{\tau}
$$

Where

$$
\begin{aligned}
& \boldsymbol{D}_{r e q}=\boldsymbol{D}_{r r}-\boldsymbol{D}_{r f} \boldsymbol{D}_{f f}^{-1} \boldsymbol{D}_{f r} . \\
& \boldsymbol{C}_{r e q}=-\boldsymbol{D}_{r f} \boldsymbol{D}_{f f}^{-1} \boldsymbol{C}_{f r} \dot{\boldsymbol{\theta}}-\boldsymbol{D}_{r f} \boldsymbol{D}_{f f}^{-1} \boldsymbol{C}_{f f} \dot{\boldsymbol{\eta}}- \\
& \boldsymbol{D}_{r f} \boldsymbol{D}_{f f}^{-1} \boldsymbol{K} \boldsymbol{\eta}+\boldsymbol{C}_{r r} \dot{\boldsymbol{\theta}}+\boldsymbol{C}_{r f} \dot{\boldsymbol{\eta}} .
\end{aligned}
$$

If the flexible space manipulator model is accurately known, then the following control law designed according to the calculation moment method is obtained:

$\boldsymbol{\tau}=\boldsymbol{D}_{r e q}\left(\ddot{\boldsymbol{\theta}}_{d}-\boldsymbol{k}_{v} \dot{\boldsymbol{e}}-\boldsymbol{k}_{p} \boldsymbol{e}\right)+\boldsymbol{C}_{r e q}$

Subsequently, a closed-loop system could be achieved:

$$
\ddot{\boldsymbol{e}}+\boldsymbol{k}_{v} \dot{\boldsymbol{e}}+\boldsymbol{k}_{p} \boldsymbol{e}=0
$$

where $\boldsymbol{\theta}_{d}=\left(\begin{array}{lll}\theta_{0 d} & \theta_{1 d} & \theta_{2 d}\end{array}\right)^{\mathrm{T}}$ is the rigid motion (the desired motion trajectory of the base's attitude and joint's angles). $\dot{\boldsymbol{\theta}}_{d}$ and $\ddot{\boldsymbol{\theta}}_{d}$ are the corresponding desired speed and accelerated speed: $\boldsymbol{e}=\boldsymbol{\theta}-\boldsymbol{\theta}_{d}$ and $\dot{\boldsymbol{e}}=\dot{\boldsymbol{\theta}}-\dot{\boldsymbol{\theta}}_{d} . \boldsymbol{k}_{v}$ and $\boldsymbol{k}_{p}$ are the symmetric and positive defined constant matrixes. The trajectory tracking of the rigid motion of the flexible space manipulator system could be realized by choosing $\boldsymbol{k}_{v}$ and $\boldsymbol{k}_{p}$ appropriately.

Nevertheless, developing an accurate model of the flexible space manipulator system in practical engineering is difficult. Instead, only a nominal model can be achieved. Suppose the nominal models of the system are expressed as
$\boldsymbol{D}_{0}$ and $\boldsymbol{C}_{0}$. Then, the nominal models are used to design the control laws of the system as follows

$$
\boldsymbol{\tau}=\boldsymbol{D}_{0}\left(\ddot{\boldsymbol{\theta}}_{d}-\boldsymbol{k}_{v} \dot{\boldsymbol{e}}-\boldsymbol{k}_{p} \boldsymbol{e}\right)+\boldsymbol{C}_{0}
$$

Consequently,

$$
\boldsymbol{D}_{r e q} \ddot{\boldsymbol{\theta}}+\boldsymbol{C}_{r e q}=\boldsymbol{D}_{0}\left(\ddot{\boldsymbol{\theta}}_{d}-\boldsymbol{k}_{v} \dot{\boldsymbol{e}}-\boldsymbol{k}_{p} \boldsymbol{e}\right)+\boldsymbol{C}_{0}
$$

Let $\boldsymbol{\Delta D}=\boldsymbol{D}_{\text {req }}-\boldsymbol{D}_{0}$ and $\boldsymbol{\Delta C}=\boldsymbol{C}_{\text {req }}-\boldsymbol{C}_{0}$. The following closedloop system can be obtained:

$$
\ddot{\boldsymbol{e}}+\boldsymbol{k}_{v} \dot{\boldsymbol{e}}+\boldsymbol{k}_{p} \boldsymbol{e}=\boldsymbol{D}_{0}^{-1}(\Delta D \ddot{\theta}+\Delta C)
$$

Obviously, the model uncertainty of the flexible space manipulator system will decrease tracking performances. The goal of this study is to design a compensation control unit to improve the trajectory tracking performances.

Let $\boldsymbol{x}=(\boldsymbol{e}, \dot{\boldsymbol{e}})^{\mathrm{T}}$ and $\boldsymbol{f}=\boldsymbol{D}_{0}^{-1}(\boldsymbol{\Delta} \boldsymbol{D} \ddot{\boldsymbol{\theta}}+\boldsymbol{\Delta} \boldsymbol{C})$ represent the model uncertainty. Then, the following state equation can be gained:

$$
\dot{x}=A x+B f
$$

Where

$$
\boldsymbol{A}=\left[\begin{array}{rr}
0 & \boldsymbol{I} \\
-\boldsymbol{k}_{v} & -\boldsymbol{k}_{p}
\end{array}\right], \boldsymbol{B}=\left[\begin{array}{l}
0 \\
\boldsymbol{I}
\end{array}\right] .
$$

If $\boldsymbol{f}$ is known, then the designed and corrected control law of the calculation moment is

$$
\boldsymbol{\tau}=\boldsymbol{D}_{0}\left(\ddot{\boldsymbol{\theta}}_{d}-\boldsymbol{k}_{v} \dot{\boldsymbol{e}}-\boldsymbol{k}_{p} \boldsymbol{e}\right)+\boldsymbol{C}_{0}-\boldsymbol{D}_{0} \boldsymbol{f}
$$

Therefore, the closed-loop system can be gained from the Eq. (7) and can compensate for model uncertainty well. However, the model uncertainty term $(f)$ in practice is unknown, and thus the modified control law of Eq.(12) is inapplicable. Nevertheless, the RBF neural network is a feedback network with good nonlinear approximation ability and no local minimum. The RBF neural network is also convenient for mathematical processing and has high convergence speed. Therefore, said network was applied for the approximation of $\boldsymbol{f}$.

The relationship between the input and output of a RBF neural network which has $l$ inputs, $m$ units of the hidden layer, and $n$ outputs can be expressed as

$$
\boldsymbol{y}=\boldsymbol{w}^{\mathrm{T}} \boldsymbol{\alpha}(\boldsymbol{X})
$$

where $\quad \boldsymbol{X}=\left[\begin{array}{llll}X_{1} & X_{2} & \cdots & X_{l}\end{array}\right]^{\mathrm{T}}$ is an input vector, $\boldsymbol{w}^{\mathrm{T}}=\left[w_{i j}\right](i=1, \cdots, n ; j=1, \cdots, m)$ is the network weight matrix, $\boldsymbol{\alpha}(X)=\left[\begin{array}{lll}\alpha_{1}(X) & \cdots & \alpha_{m}(X)\end{array}\right]^{\mathrm{T}}$ is the column vector of the basis function, and $\boldsymbol{y}=\left[\begin{array}{llll}y_{1} & y_{2} & \cdots & y_{n}\end{array}\right]^{\mathrm{T}}$ is the output column vector.

In Eq.(13), the elements of $\boldsymbol{y}=\left[\begin{array}{llll}y_{1} & y_{2} & \cdots & y_{n}\end{array}\right]^{\mathrm{T}}$ constitute the Gaussian basis function and

$\alpha_{j}(X)=\exp \left(-\left\|\boldsymbol{X}-\boldsymbol{c}_{j}\right\|^{2} / \sigma_{j}^{2}\right)$ 
where $\boldsymbol{c}_{j}$ is the center vector of the basis function $j$ and $\sigma_{j}$ is the width of the basis function $j$.

The expression of $\boldsymbol{f}$ clearly indicates that it is a nonlinear function of $\boldsymbol{\theta}, \dot{\boldsymbol{\theta}}, \ddot{\boldsymbol{\theta}}$ and $\boldsymbol{\eta}, \dot{\boldsymbol{\eta}}$. If the input of the neural network is $\boldsymbol{X}=\left[\begin{array}{lllll}\boldsymbol{\theta}^{\mathrm{T}} & \dot{\boldsymbol{\theta}}^{\mathrm{T}} & \ddot{\boldsymbol{\theta}}^{\mathrm{T}} & \boldsymbol{\eta}^{\mathrm{T}} & \dot{\boldsymbol{\eta}}^{\mathrm{T}}\end{array}\right]^{\mathrm{T}}$, then the RBF neural network can approximate the model uncertainty consistently at any precision. Hence, an ideal output $f^{*}$ and an ideal weight $\boldsymbol{w}^{*}$ exists to meet

$$
\boldsymbol{f}(\boldsymbol{X})=\boldsymbol{f}^{*}(\boldsymbol{X})+\boldsymbol{\varepsilon}=\boldsymbol{w}^{* \mathrm{~T}} \boldsymbol{\alpha}(\boldsymbol{X})+\boldsymbol{\varepsilon}
$$

where $\varepsilon$ is an approximation error. If $\varepsilon$ bounded, then the boundary is $\varepsilon_{0}$.

The practical output of the RBF neural network $(\hat{\boldsymbol{f}})$ was used to for approximation of $\boldsymbol{f}$.

$$
\hat{f}(X)=\hat{\boldsymbol{w}}^{\mathrm{T}} \boldsymbol{\alpha}(\boldsymbol{X})
$$

where $\hat{\boldsymbol{w}}$ is the estimated value of $\boldsymbol{w}^{*}$.

Hence, the control input law in the following form is designed:

$$
\boldsymbol{\tau}=\boldsymbol{D}_{0}\left(\ddot{\boldsymbol{\theta}}_{d}-\boldsymbol{k}_{v} \dot{\boldsymbol{e}}-\boldsymbol{k}_{p} \boldsymbol{e}\right)+\boldsymbol{C}_{0}-\boldsymbol{D}_{0} \hat{\boldsymbol{f}}
$$

The control law (Eq.[17]) is introduced into Eq.(5), so the following closed-loop system is obtained:

$$
\begin{array}{r}
\dot{\boldsymbol{x}}=\boldsymbol{A} \boldsymbol{x}+\boldsymbol{B}\left[\boldsymbol{w}^{* \mathrm{~T}} \boldsymbol{\alpha}(\boldsymbol{X})+\boldsymbol{\varepsilon}-\hat{\boldsymbol{w}}^{\mathrm{T}} \boldsymbol{\alpha}(\boldsymbol{X})\right] \\
=\boldsymbol{A} \boldsymbol{x}+\boldsymbol{B}\left[-\tilde{\boldsymbol{w}}^{\mathrm{T}} \boldsymbol{\alpha}(\boldsymbol{X})+\boldsymbol{\varepsilon}\right]
\end{array}
$$

where $\tilde{\boldsymbol{w}}=\hat{\boldsymbol{w}}-\boldsymbol{w}^{*}$.

The following positive definition function $(V)$ was chosen as the quasi-Lyapunov function:

$$
V=1 / 2 \boldsymbol{x}^{\mathrm{T}} \boldsymbol{P} \boldsymbol{x}+1 /(2 \gamma)\|\tilde{\boldsymbol{w}}\|^{2} \quad(\gamma>0)
$$

where $\|\boldsymbol{R}\|$ is the Frobenius norm of a matrix and is defined as

$$
\|\boldsymbol{R}\|^{2}=\sum_{i, j}\left|R_{i j}\right|^{2}=\operatorname{tr}\left(\boldsymbol{R} \boldsymbol{R}^{\mathrm{T}}\right)=\operatorname{tr}\left(\boldsymbol{R}^{\mathrm{T}} \boldsymbol{R}\right)
$$

$\boldsymbol{P}$ is the symmetric and positive definition matrix and meets the following Lyapunov equation:

$$
\boldsymbol{P} \boldsymbol{A}+\boldsymbol{A}^{\mathrm{T}} \boldsymbol{P}=-\boldsymbol{Q} \quad(\boldsymbol{Q}>0)
$$

We calculate the time differential of $V$ and it can get from the Eq.(18) that

$$
\begin{aligned}
& V= 1 / 2\left[\boldsymbol{x}^{\mathrm{T}} \boldsymbol{P} \dot{\boldsymbol{x}}+\dot{\boldsymbol{x}}^{\mathrm{T}} \boldsymbol{P} \boldsymbol{x}\right]+1 / \gamma \operatorname{tr}\left(\dot{\tilde{\boldsymbol{w}}}^{\mathrm{T}} \tilde{\boldsymbol{w}}\right) \\
&= 1 / 2\left[\boldsymbol{x}^{\mathrm{T}}\left(\boldsymbol{P} \boldsymbol{A}+\boldsymbol{A}^{\mathrm{T}} \boldsymbol{P}\right) \boldsymbol{x}\right]+ \\
& {\left[-\tilde{\boldsymbol{w}}^{\mathrm{T}} \boldsymbol{\alpha}(\boldsymbol{X})+\boldsymbol{\varepsilon}\right]^{\mathrm{T}} \boldsymbol{B}^{\mathrm{T}} \boldsymbol{P} \boldsymbol{x}+1 / \gamma \operatorname{tr}\left(\dot{\tilde{\boldsymbol{w}}}^{\mathrm{T}} \tilde{\boldsymbol{w}}\right) } \\
&=-1 / 2 \boldsymbol{x}^{\mathrm{T}} \boldsymbol{Q} \boldsymbol{x}-\boldsymbol{\alpha}(\boldsymbol{X})^{\mathrm{T}} \tilde{\boldsymbol{w}} \boldsymbol{B}^{\mathrm{T}} \boldsymbol{P} \boldsymbol{x}+ \\
& \boldsymbol{\varepsilon}^{\mathrm{T}} \boldsymbol{B}^{\mathrm{T}} \boldsymbol{P} \boldsymbol{x}+1 / \gamma \operatorname{tr}\left(\dot{\tilde{\boldsymbol{w}}}^{\mathrm{T}} \tilde{\boldsymbol{w}}\right) .
\end{aligned}
$$

$$
\boldsymbol{\alpha}(\boldsymbol{X})^{\mathrm{T}} \tilde{\boldsymbol{w}} \boldsymbol{B}^{\mathrm{T}} \boldsymbol{P} \boldsymbol{x}=\operatorname{tr}\left(\boldsymbol{B}^{\mathrm{T}} \boldsymbol{P} \boldsymbol{x} \boldsymbol{\alpha}(\boldsymbol{X})^{\mathrm{T}} \tilde{\boldsymbol{w}}\right)
$$

Then,

$$
\begin{aligned}
\dot{V}= & -1 / 2 \boldsymbol{x}^{\mathrm{T}} \boldsymbol{Q} \boldsymbol{x}+\boldsymbol{\varepsilon}^{\mathrm{T}} \boldsymbol{B}^{\mathrm{T}} \boldsymbol{P} \boldsymbol{x}+ \\
& 1 / \gamma \operatorname{tr}\left[-\gamma \boldsymbol{B}^{\mathrm{T}} \boldsymbol{P} \boldsymbol{x} \boldsymbol{\alpha}(\boldsymbol{X})^{\mathrm{T}} \tilde{\boldsymbol{w}}+\dot{\tilde{\boldsymbol{w}}}^{\mathrm{T}} \tilde{\boldsymbol{w}}\right]
\end{aligned}
$$

If the weighted adapted updating law is selected,

$$
\dot{\hat{\boldsymbol{w}}}=\gamma \boldsymbol{\alpha}(\boldsymbol{X}) \boldsymbol{x}^{\mathrm{T}} \boldsymbol{P B}
$$

Then,

$$
\dot{V}=-1 / 2 \boldsymbol{x}^{\mathrm{T}} \boldsymbol{Q} \boldsymbol{x}+\boldsymbol{\varepsilon}^{\mathrm{T}} \boldsymbol{B}^{\mathrm{T}} \boldsymbol{P} \boldsymbol{x}
$$

Observe that $\left\|\varepsilon^{\mathrm{T}}\right\| \leq\left\|\boldsymbol{\varepsilon}_{0}\right\|$ and $\|\boldsymbol{B}\|=1$. Therefore, we can obtain

$$
\begin{aligned}
\dot{V} \leq-1 / 2 \lambda_{\text {min }}(\boldsymbol{Q})\|\boldsymbol{x}\|^{2}+\left\|\boldsymbol{\varepsilon}_{0}\right\| \lambda_{\text {max }}(\boldsymbol{P})\|\boldsymbol{x}\| \\
=-1 / 2\|\boldsymbol{x}\|\left[\lambda_{\text {min }}(\boldsymbol{Q})\|\boldsymbol{x}\|-2\left\|\boldsymbol{\varepsilon}_{0}\right\| \lambda_{\text {max }}(\boldsymbol{P})\right]
\end{aligned}
$$

where $\lambda_{\min }(\boldsymbol{Q})$ is the minimum eigenvalue of the matrix $\boldsymbol{Q}$ and $\lambda_{\min }(\boldsymbol{P})$ is the minimum eigenvalue of the matrix $\boldsymbol{P}$.

Eq. (27) indicates that $\lambda_{\text {min }}(\boldsymbol{Q}) \geq 2\left\|\boldsymbol{\varepsilon}_{0}\right\| \lambda_{\text {max }}(\boldsymbol{P}) /\|\boldsymbol{x}\|$ is required to achieve $\dot{V} \leq 0$. In other words, the convergence radius of $\boldsymbol{x}$ is $2\left\|\varepsilon_{0}\right\| \lambda_{\max }(\boldsymbol{P}) / \lambda_{\text {min }}(\boldsymbol{Q})$.

\subsection{Control scheme on the basis of the hybrid trajectory}

The neural network control scheme according to model uncertainty compensation designed in the previous section can control the flexible space manipulator to finish the rigid motion (trajectory tracking of the base's attitude and joint's angles) but cannot suppress the vibration of the flexible link. Accordingly, the concept of virtual control force was used to generate a hybrid trajectory that can reflect both the flexible vibration modal and rigid motion trajectory to stabilize the vibration of the flexible link.

To use the concept of virtual control force, the error ( $\left.\boldsymbol{e}_{h}=\boldsymbol{\theta}_{h}-\boldsymbol{\theta}_{d}\right)$ between the hybrid trajectory $\boldsymbol{\theta}_{h}=\left(\begin{array}{lll}\theta_{0 h} & \theta_{1 h} & \theta_{2 h}\end{array}\right)^{\mathrm{T}}$ and the desired motion trajectory must be defined. In this part, $\boldsymbol{e}_{h}$ is generated by the following second-order command generator:

$$
\ddot{\boldsymbol{e}}_{h}+\boldsymbol{a} \dot{\boldsymbol{e}}_{h}+\boldsymbol{b} \boldsymbol{e}_{h}=\boldsymbol{F}
$$

where $\boldsymbol{a}$ and $\boldsymbol{b}$ are the diagonal matrixes of the positive definite constant. $\boldsymbol{F}$ is the virtual control force vector and can stabilize the flexible vibration and maintain robustness of the compensation control of the rigid motion neural network. The expression is given in the following text.

Eq. (17) is rewritten and the control law based on the hybrid trajectory is designed as follows:

$$
\boldsymbol{\tau}=\boldsymbol{D}_{0}\left(\ddot{\boldsymbol{\theta}}_{h}-\boldsymbol{k}_{v} \dot{\boldsymbol{e}}_{r}-\boldsymbol{k}_{p} \boldsymbol{e}_{r}\right)+\boldsymbol{C}_{0}-\boldsymbol{D}_{0} \hat{\boldsymbol{f}}
$$

Note that 
where $\boldsymbol{e}_{r}=\boldsymbol{\theta}-\boldsymbol{\theta}_{h}$ is the error between the hybrid trajectory and practical trajectory, and $\dot{\boldsymbol{e}}_{r}=\dot{\boldsymbol{\theta}}-\dot{\boldsymbol{\theta}}_{h}$. Eq. (29) is brought into Eq.(5) and the error equation of $\boldsymbol{e}_{\mathrm{r}}$ can be obtained:

$$
\ddot{\boldsymbol{e}}_{r}+\boldsymbol{k}_{v} \dot{\boldsymbol{e}}_{r}+\boldsymbol{k}_{p} \boldsymbol{e}_{r}=\boldsymbol{D}_{0} \boldsymbol{\varepsilon}
$$

Eq.(30) is brought into Eq.(28), and the error equation of the tracking error $\boldsymbol{e}$ can be obtained:

$$
\ddot{\boldsymbol{e}}+\boldsymbol{a} \dot{\boldsymbol{e}}+\boldsymbol{b} \boldsymbol{e}=\boldsymbol{F}+\boldsymbol{H}_{D_{0} \varepsilon} \boldsymbol{D}_{0} \boldsymbol{\varepsilon}
$$

where

$$
\boldsymbol{H}_{D_{0} \varepsilon}=\operatorname{diag}\left(\boldsymbol{H}_{D_{0} \varepsilon 1}, \boldsymbol{H}_{D_{0} \varepsilon}, \boldsymbol{H}_{D_{0} \varepsilon 3}\right)
$$

$$
\boldsymbol{H}_{D_{0} \varepsilon i}(j \omega)=\frac{-\omega^{2}+j \omega a_{i}+b_{i}}{-\omega^{2}+j \omega k_{v i}+k_{p i}}(i=1,2,3) \text {. }
$$

$\ddot{\boldsymbol{\theta}}$ can be calculated from Eq.(31):

$$
\ddot{\boldsymbol{\theta}}=\ddot{\boldsymbol{\theta}}_{d}-\boldsymbol{a} \dot{\boldsymbol{e}}-\boldsymbol{b} \boldsymbol{e}+\boldsymbol{F}+\boldsymbol{H}_{D_{0} \varepsilon} \boldsymbol{D}_{0} \boldsymbol{\varepsilon}
$$

Eq.(3) indicates that the nominal model of the flexible vibration can be written as:

$$
\hat{\boldsymbol{D}}_{f r} \ddot{\boldsymbol{\theta}}+\hat{\boldsymbol{D}}_{f f} \ddot{\boldsymbol{\eta}}+\hat{\boldsymbol{C}}_{f r} \dot{\boldsymbol{\theta}}+\hat{\boldsymbol{C}}_{f f} \dot{\boldsymbol{\eta}}+\hat{\boldsymbol{K}} \boldsymbol{\eta}=0
$$

where the matrix with a superscript “^” represents the corresponding nominal model.

Eq.(33) is brought into Eq.(32), and $\ddot{\boldsymbol{\eta}}$ can be solved as follows:

$$
\begin{aligned}
\ddot{\boldsymbol{\eta}}=-\hat{\boldsymbol{D}}_{f f}^{-1} & \hat{\boldsymbol{C}}_{f f} \dot{\boldsymbol{\eta}}-\hat{\boldsymbol{D}}_{f f}^{-1} \hat{\boldsymbol{K}} \boldsymbol{\eta}+\hat{\boldsymbol{D}}_{f f}^{-1} \hat{\boldsymbol{D}}_{f r}(\boldsymbol{a} \dot{\boldsymbol{e}}+\boldsymbol{b} \boldsymbol{e})- \\
& \hat{\boldsymbol{D}}_{f f}^{-1} \hat{\boldsymbol{D}}_{f r} \boldsymbol{F}-\hat{\boldsymbol{D}}_{f f}^{-1}\left(\hat{\boldsymbol{D}}_{f r} \ddot{\boldsymbol{\theta}}_{d}+\hat{\boldsymbol{C}}_{f r} \dot{\boldsymbol{\theta}}+\hat{\boldsymbol{D}}_{f r} \boldsymbol{H}_{D_{0} \varepsilon} \boldsymbol{D}_{0} \boldsymbol{\varepsilon}\right)
\end{aligned}
$$

Eqs.(31) and (34) reveal that the error state equation containing the tracking error and flexible modal is

$$
\dot{z}=c z+d F+E
$$

Where

$$
\begin{aligned}
\boldsymbol{z}=\left[\begin{array}{llll}
\boldsymbol{\eta}^{\mathrm{T}} & \boldsymbol{e}^{\mathrm{T}} & \dot{\boldsymbol{\eta}}^{\mathrm{T}} & \dot{\boldsymbol{e}}^{\mathrm{T}}
\end{array}\right]^{\mathrm{T}}, \\
\boldsymbol{c}=\left[\begin{array}{cccc}
\boldsymbol{0} & \boldsymbol{0} & \boldsymbol{I} & \boldsymbol{0} \\
\boldsymbol{0} & \boldsymbol{0} & \boldsymbol{0} & \boldsymbol{I} \\
-\hat{\boldsymbol{D}}_{f f}^{-1} \hat{\boldsymbol{K}} & \hat{\boldsymbol{D}}_{f f}^{-1} \hat{\boldsymbol{D}}_{f r} \boldsymbol{b} & -\hat{\boldsymbol{D}}_{f f}^{-1} \hat{\boldsymbol{C}}_{f f} & \hat{\boldsymbol{D}}_{f f}^{-1} \hat{\boldsymbol{D}}_{f r} \boldsymbol{a} \\
\boldsymbol{0} & -\boldsymbol{b} & \boldsymbol{0} & -\boldsymbol{a}
\end{array}\right], \\
\boldsymbol{d}=\left[\begin{array}{c}
\boldsymbol{0} \\
\boldsymbol{0} \\
-\hat{\boldsymbol{D}}_{f f}^{-1} \hat{\boldsymbol{D}}_{f r} \\
\boldsymbol{I}
\end{array}\right], \\
\boldsymbol{E}=\left[\begin{array}{c}
\boldsymbol{0} \\
-\hat{\boldsymbol{D}}_{f f}^{-1}\left(\hat{\boldsymbol{D}}_{f r} \ddot{\boldsymbol{\theta}}_{d}+\hat{\boldsymbol{C}}_{f r} \dot{\boldsymbol{\theta}}+\hat{\boldsymbol{D}}_{f r} \boldsymbol{H}_{D_{0} \varepsilon} \boldsymbol{D}_{0} \boldsymbol{\varepsilon}\right) \\
\boldsymbol{H}_{D_{0} \varepsilon} \boldsymbol{D}_{0} \boldsymbol{\varepsilon}
\end{array}\right] .
\end{aligned}
$$

Now, the optical LQR was used to design a hybrid controller. For this purpose, $\boldsymbol{E}$ was viewed as a disturbance and the performance index function of the LQR was introduced in

$$
J=\frac{1}{2} \int_{0}^{\infty}\left(\boldsymbol{z}^{\mathrm{T}} \boldsymbol{q} \boldsymbol{z}+\boldsymbol{F}^{\mathrm{T}} \boldsymbol{r} \boldsymbol{F}\right) \mathrm{d} t
$$

where $\boldsymbol{q}$ and $\boldsymbol{r}$ are the positive definite symmetric constant matrixes. The performance index function in this form brings relatively high accuracy and a slight energy consumption of the control system. Moreover, the function can effectively suppress the vibration generated by the flexible link.

According to the optical LQR theory, to minimize $J$, the virtual control force should be

$$
\boldsymbol{F}=-\boldsymbol{r}^{-1} \boldsymbol{d}^{\mathrm{T}} \boldsymbol{G} \boldsymbol{z}
$$

where $\boldsymbol{G}$ meets the following Riccat algebraic equation:

$$
\boldsymbol{G} \boldsymbol{c}+\boldsymbol{c}^{\mathrm{T}} \boldsymbol{G}-\boldsymbol{G} \boldsymbol{d} \boldsymbol{r}^{-1} \boldsymbol{d}^{\mathrm{T}} \boldsymbol{G}+\boldsymbol{q}=0
$$

In this way, the closed-loop system (35) is changed into

$$
\dot{\boldsymbol{z}}=\left(\boldsymbol{c}-\boldsymbol{d} \boldsymbol{r}^{-1} \boldsymbol{d}^{\mathrm{T}} \boldsymbol{G}\right) \boldsymbol{z}+\boldsymbol{E}
$$

At this moment, if $\boldsymbol{E}=0$, then the closed-loop system is approximately stable.

In the following text, the full condition for the approximate stability of the closed-loop system when $\boldsymbol{E} \neq 0$ is proposed. Let $\boldsymbol{N}=2 \boldsymbol{q}+\boldsymbol{c}^{\mathrm{T}} \boldsymbol{G}+\boldsymbol{G} \boldsymbol{c}$ and $\|\boldsymbol{R}\|_{S},\|\boldsymbol{R}\|_{E}$, $\max \lambda(\boldsymbol{R})$ and $\min \lambda(\boldsymbol{R})$ be the spectral norm $\left(\max \left[\lambda\left(\boldsymbol{R} \boldsymbol{R}^{\mathrm{T}}\right)\right]^{1 / 2}\right)$, the Euclidean norm $\left(\|\boldsymbol{R}\|^{2}=\left(\sum_{i, j}\left|R_{i j}\right|^{2}\right)^{1 / 2}\right)$, and maximum eigenvalue and minimum eigenvalue of the matrix $\boldsymbol{R}$, respectively. If the nonlinear function $\boldsymbol{E}$ meets the following conditions:

$\frac{\|\boldsymbol{E}\|_{E}}{\|\boldsymbol{z}\|_{E}} \leq \boldsymbol{\mu} \equiv \frac{1}{2\left\|\boldsymbol{N}^{-1}\right\|_{S}\|\boldsymbol{G}\|_{S}}=\frac{\min \lambda(\boldsymbol{N})}{2 \max \lambda(\boldsymbol{G})}$

Then, the closed-loop system (Eq.[39]) is stable.

\section{Result Analysis and Discussion}

The free-floating flexible space manipulator system which generates the planar motion is shown in Fig.1. The neural network control scheme based on the hybrid trajectory (Eq.[29]) and the weighted adaptive updating law (Eq.[25]) were used in the numerical simulation test of the system.

During the simulation, the relevant inertial parameters of the system are hypothesized to include $m_{0}=40 \mathrm{~kg}, l_{0}=1.5 \mathrm{~m}$, and $J_{0}=35 \mathrm{~kg} \cdot \mathrm{m}^{2}$. The parameters of the rigid link are $m_{1}=2 \mathrm{~kg}$, $l_{1}=2 \mathrm{~m}, a_{1}=1 \mathrm{~m}$ and $J_{1}=2 \mathrm{~kg} \cdot \mathrm{m}^{2}$. The parameters of the flexible link are $l_{2}=1 \mathrm{~m}, \rho=1 \mathrm{~kg} / \mathrm{m}$, and $E I=40 \mathrm{~Pa}$. Moreover, nominal models of the system are $\boldsymbol{D}_{0}=0.9 \boldsymbol{D}_{\text {req }}$ and $\boldsymbol{C}_{0}=0.9 \boldsymbol{C}_{\text {req }}$, respectively.

The desired motion trajectories of the base's attitude and two joints' angles of the flexible space manipulator are 
$\theta_{0 d}=0$,

$\theta_{1 d}=\cos (\pi \mathrm{t} / 3)+\pi / 3$

$\theta_{2 d}=\sin (\pi \mathrm{t} / 3)-\pi / 3$ (unit: $\left.\mathrm{rad}\right)$.

The initial values of the attitude angle of the base and joint angle of the arm are $\theta_{0}(0)=0.1 \mathrm{rad}, \theta_{1}(0)=1.5 \mathrm{rad}$, and $\theta_{2}(0)=-0.5 \mathrm{rad}$. The entire tracking process takes $t=8 \mathrm{~s}$.

Simulation parameters were chosen as follows: $\boldsymbol{k}_{v}=\operatorname{diag}[3], \quad \boldsymbol{k}_{p}=\operatorname{diag}[1], \quad \gamma=20, \quad \boldsymbol{Q}=\operatorname{diag}[50], \quad \boldsymbol{a}=\operatorname{diag}[1]$, $\boldsymbol{b}=\operatorname{diag}[50], \boldsymbol{q}=\operatorname{diag}[1]$, and $\boldsymbol{r}=\operatorname{diag}[1]$.

Simulation results are shown in the following figures. The comparison between the practical motion trajectory of base's attitude of the flexible space manipulator (solid line) and the desired motion trajectory (dotted line) is shown in Fig.2. The comparison between the practical motion trajectory of the two hinge joints of the flexible space manipulator (solid line) and the desired motion trajectory (dotted line) is shown in Fig.3 The simulation results indicate that when model uncertainty of system exists, the errors of base's attitude angle as well as errors of the practical trajectories of joint angles 1 and 2 of the manipulator are $5 \times 10^{-4} \mathrm{rad}, 7.5 \times 10^{-3} \mathrm{rad}$ and $2 \times 10^{-3} \mathrm{rad}$, respectively and at approximately $4 \mathrm{~s}$ later. This outcome suggest that the compensation controller of RBF neural network can effectively control the base's attitude and joint's angles of the flexible space manipulator to accurately track the desired motion trajectory.

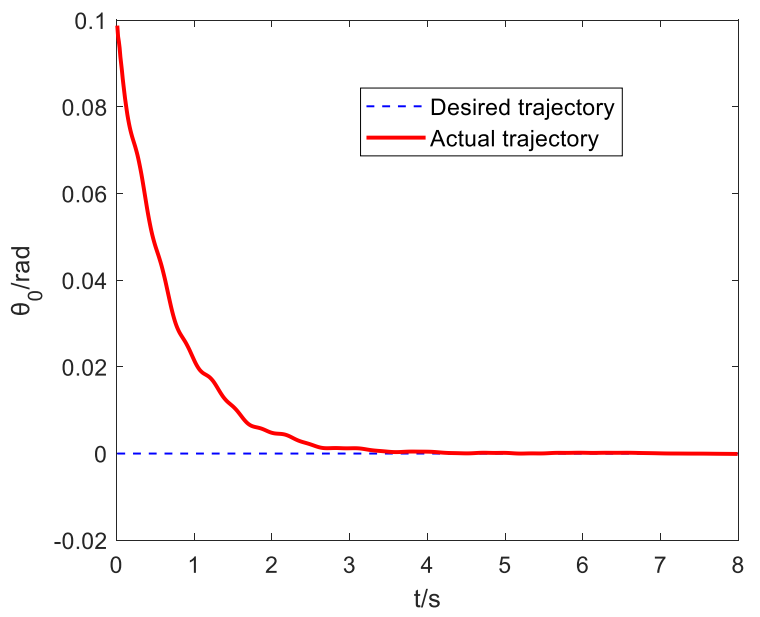

Fig. 2. The actual and desired trajectory of the base's attitude

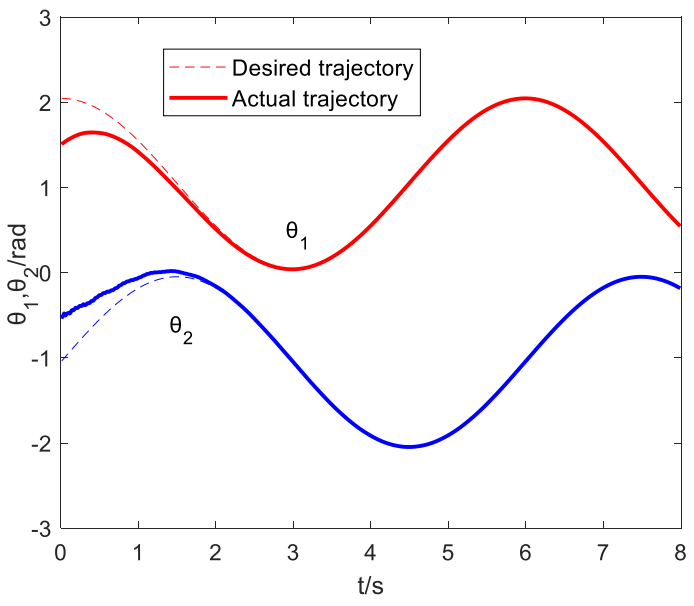

Fig. 3. The actual and desired trajectory of the two hinge joints

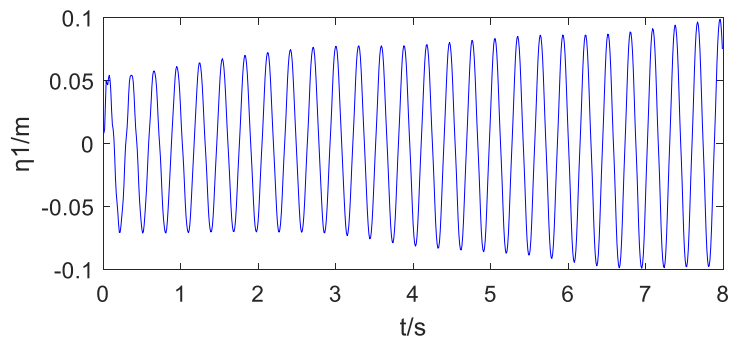

Fig. 4. The first-order modal of flexible link (neural network compensation control)

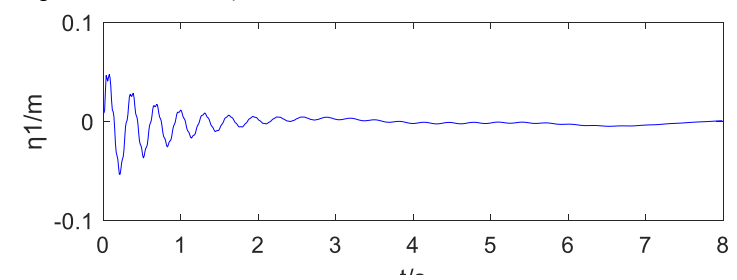

Fig. 5. The first-order modal of flexible link (hybrid trajectory control)

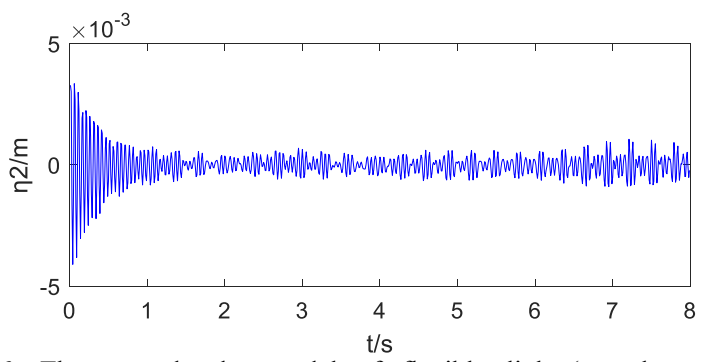

Fig.6. The second-order modal of flexible link (neural network compensation control)

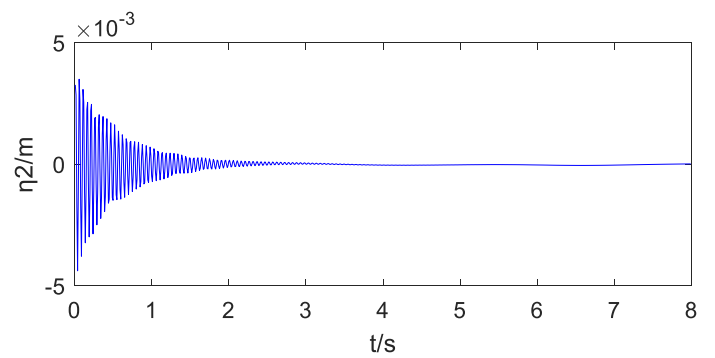

Fig. 7. The second-order modal of flexible link (hybrid trajectory control)

Simulation results of the first-order and second-order modals of the flexible link when only the neural network compensation control (Eq. [17]) is applied are shown in Figs. 4 and 6, respectively. Simulation results of the firstorder and second-order modals of the flexible link when the control is based on the hybrid trajectory (Eq.[29]) are shown in Figs.5 and 7. Under neural network compensation control, $\eta_{1}$ vibrates in the range of $\pm 0.1 \mathrm{~m}$ and $\eta_{2}$ vibrates in the range of $\pm 0.001 \mathrm{~m}$ after approximately $7 \mathrm{~s}$. Under the control based on the hybrid trajectory, $\eta_{1}$ attenuates from $-0.05 \mathrm{~m}$ at the initial $0.2 \mathrm{~s}$ to $0.002 \mathrm{~m}$, while $\eta_{2}$ attenuates from $-0.0044 \mathrm{~m}$ at the initial $0.04 \mathrm{~s}$ to $5 \times 10^{-5} \mathrm{~m}$. A summary of these four modal diagrams is shown in Table 1. According to a comparative analysis, the control scheme based on a hybrid trajectory can suppress the vibration of the flexible link of the space manipulator more effectively and basically eliminates the vibration of the flexible link relative to the control scheme based on the desired trajectory and using neural network compensation control only, thereby enabling the increase of the trajectory tracking accuracy of the flexible space manipulator. 
Table 1. Comparison for modals of flexible link

\begin{tabular}{l|c|c}
\hline Control scheme & $\eta_{1}(\mathrm{~m})$ & $\eta_{2}(\mathrm{~m})$ \\
\hline Neural network compensation control & 0.1 & 0.001 \\
hybrid trajectory control & 0.002 & 0.00005 \\
\hline
\end{tabular}

According to above simulation results, the proposed neural network compensation control scheme based on a hybrid trajectory needs neither an accurate dynamics model of the system nor a linear relationship between the dynamic equation of the system and the inertia parameters. The proposed method can well compensate for the negative impacts on the trajectory tracking based on model uncertainty. The proposed control scheme not only maintains the robustness of the original scheme to model uncertainty, but can also actively suppress flexible vibration. The technique realizes high-accuracy trajectory tracking of the flexible manipulator.

\section{Conclusions}

To avoid the influences of model uncertainty and suppress the vibration of a flexible manipulator and realize highaccuracy trajectory tracking, a dynamics model of the flexible space manipulator system with an attitudecontrolled base is constructed. On this basis, a neural network control scheme based on hybrid trajectory is applied to study the rigid motion and flexible vibration suppression of the system. Some major conclusions could be drawn:

1) The proposed control scheme compensates for model uncertainty by employing a neural network. It makes full use of the existing nominal model of the system. Moreover, the neural network employs online training based on the Lyapunov theory, a feature which assures convergence of the learning algorithm.
2) The virtual control force is obtained by using optimal LQR, and a hybrid trajectory that simultaneously reflects the flexible vibration modal and rigid motion trajectory is designed. The neural network control scheme based on the desired trajectory is reconstructed into a control scheme based on hybrid trajectory. It not only assures robustness of the trajectory tracking control, but also inhibits flexible vibration.

3) According to the simulation results, the base's attitude and joint's angles of the flexible space manipulator can track the desired trajectories coordinately. Compared with the neural network compensation control scheme based on the desired trajectory, the control scheme based on the hybrid trajectory can suppress flexible vibration significantly and improves trajectory tracking performances.

This study provides an effective solution for the highaccuracy trajectory tracking control of a flexible space manipulator with model uncertainty. It has important theoretical significance. However, the proposed model of the flexible link is relatively simple and does not apply other parameters like high-order modals to establish a more detailed flexible link model. Future studies can design trajectory tracking and active vibration suppression control schemes of the space manipulator for different types of flexible link models.

\section{Acknowledgements}

The authors are grateful for the support provided by the Fujian Provincial Natural Science Foundation of China (2020J01842).

This is an Open Access article distributed under the terms of the Creative Commons Attribution License.

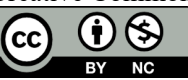

\section{References}

1. Jing Z., Qiao L., Pan H., et al., "An overview of the configuration and manipulation of soft robotics for on-orbit servicing". Science China Information Sciences, 60(050201) 2017, pp. 1-19.

2. Flores-Abad, A., Ma, O., Pham, K., Ulrich, S., "A review of space robotics technologies for on-orbit servicing". Progress in Aerospace Sciences, 68(8) 2014, pp. 1-26.

3. Larouche B P., Zhu G Z H., "Investigation of impedance controller for autonomous on-orbit servicing robot". Canadian Aeronautics \& Space Journal Le Journal Aéronautique Et Spatial Du Canada, 59(1), 2013, pp. 15-24.

4. Jing Z., Xu Q., Huang J., "A review on kinematic analysis and dynamic stable control of space flexible manipulators". Aerospace Systems, 2, 2019, pp.1-14.

5. Banerjee S., Basu S., Chaturvedi S K., et al., "A review of orbital space robots on its technical aspects". Incas Bulletin, 11(2), 2019, pp 29-43.

6. Ellery., "Tutorial Review on Space Manipulators for Space Debris Mitigation". Robotics, 8(2), 2019, pp.1-52.

7. Andrew., Long., Matthew., et al., "On-Orbit Servicing: A New Value Proposition for Satellite Design and Operation". Journal of Spacecraft and Rockets, 44(4), 2007, pp. 964-964.

8. Xu W., Liang B., Li C., et al., "Autonomous rendezvous and robotic capturing of non-cooperative target in space". Robotica, 28(5), 2010, pp. 705-718.

9. Wang Z., Zeng H., Ho D W C., et al., "Multiobjective control of a four-link flexible manipulator: a robust $\mathrm{H} \infty$ approach". IEEE Transactions on Control Systems Technology, 10(6), 2002, pp.866875.

10. Jia Y H., Hu Q., Xu S J., "Dynamics and adaptive control of a dualarm space robot with closed-loop constraints and uncertain inertial parameters". Acta Mechanica Sinica, 30(1), 2014, pp.112-124.
11. Santosha Kumar Dwivedy., Peter Eberhard., "Dynamic analysis of flexible manipulators, a literature review". Mechanism and Machine Theory, 41(7), 2006, pp. 749-777.

12. Rybus T., "Obstacle avoidance in space robotics: Review of major challenges and proposed solutions". Progress in Aerospace Sciences, 101, 2018, pp. 31-48.

13. Lou JQ., Wei YD., "Dynamic Modeling and Vibration Control of a Space Flexible Manipulator Using Piezoelectric Actuators and Sensors". Advanced Materials Research, 345, 2011, pp. 46-52.

14. Sabatini M., Gasbarri P., Monti R., Palmerini GB., "Vibration control of a flexible space manipulator during on orbit operations". Acta Astronautica, 73, 2012, pp. 109-121.

15. Zhang Q., Li C., Zhang J., Zhang J., "Smooth adaptive sliding mode vibration control of a flexible parallel manipulator with multiple smart linkages in modal space". Journal of Sound and Vibration, 411, 2017, pp. 1-19.

16. Masoudi R., Mahzoon M., "Maneuvering and Vibrations Control of a Free-Floating Space Robot with Flexible Arms". Journal of Dynamic Systems, Measurement, and Control, 133(051001), 2011, pp. $1-8$.

17. Zhang L., Liu J., "Observer-based partial differential equation boundary control for a flexible two-link manipulator in task space". IET Control Theory \& Applications, 6(13), 2012, pp. 2120-2133

18. Yang X., Ge S S., "Backstepping control and active vibration control for a free-flying space robot with rigid-flexible links by singular perturbation approach". In: 2014 IEEE International Conference on Information \& Automation, Hulun Buir, China: IEEE, 2014. pp. 164-169.

19. Yang X., Ge SS., He W., "Dynamic modelling and adaptive robust tracking control of a space robot with two-link flexible manipulators under unknown disturbances". International Journal of Control, 91(4), 2017, pp. 969-988. 
20. Su L., Hu Q., Zhang L., "Recursive Decentralized Control for Trajectory Tracking of Flexible Space Manipulators". IEEE Access, 7, 2019, pp. 39192-39206.

21. Cetin K., Tatlicioglu E., Zergeroglu E., "On Operational Space Tracking Control of Robotic Manipulators With Uncertain Dynamic and Kinematic Terms". Journal of Dynamic Systems, Measurement, and Control, 141(1), 2019, doi:10.1115/1.4041008.

22. Kumar N., "An Asymptotically Stable Control Scheme for Space Robot System". Arabian Journal for Science and Engineering, 43(12), 2018, pp. 8049-8055.
23. Yu X-Y., "Augmented robust control of a free-floating flexible space robot. Proceedings of the Institution of Mechanical Engineers". Part G: Journal of Aerospace Engineering, 229(5), 2014, pp.947-957.

24. Feng B., Ma G., Wen Q., Wang C., "Robust chattering-free sliding mode control of space robot in task space". Journal of Control Theory and Applications, 6(2), 2008, pp. 146-152.

25. Yu Z-w., Cai G-p., "Robust adaptive control of a 6-DOF space robot with flexible panels". International Journal of Dynamics and Control, 7(4), 2018, pp.1370-1378. 